\title{
Diurnal and semidiurnal internal tide energy flux at a continental slope in the South China Sea
}

\author{
Timothy F. Duda ${ }^{1}$ and Luc Rainville ${ }^{1,2}$ \\ Received 26 June 2007; revised 18 September 2007; accepted 27 November 2007; published 25 March 2008.
}

[1] Barotropic (surface) and baroclinic (internal) tides were measured at four mooring sites during a field investigation of acoustic propagation characteristics and physical oceanography in the northern South China Sea. The mooring positions were in a line moving up the shallow portion of a continental slope at water depths between 350 and $85 \mathrm{~m}$. Using time series of temperature and velocity, at several depths, 1-month series of semidiurnal and diurnal species internal tidal energy flux vectors were computed for three sites, with a 14-day series computed for the fourth (shallow) site. The internal tides had a temporal signature that was not in complete accord with the barotropic tides, showing an enhancement of diurnal internal tides with respect to semidiurnal. Bathymetric slope, barotropic tidal fluid particle trajectories, and scale of generation site versus internal tide wavelength are investigated as possible causes of the differing response of the species.

Citation: Duda, T. F., and L. Rainville (2008), Diurnal and semidiurnal internal tide energy flux at a continental slope in the South China Sea, J. Geophys. Res., 113, C03025, doi:10.1029/2007JC004418.

\section{Introduction}

[2] Internal gravity waves at or near tidal frequencies, called internal tides, have been identified throughout the oceans and observed in detail at a few locations. The generation of these waves is of interest because they are an important link in the chain of energy flux from tides to internal waves of all frequencies (including steep nonlinear internal waves) and turbulence. Here we examine data from a low-latitude site, in the northern portion of the South China Sea, where internal waves propagate as free waves at both semidiurnal and diurnal frequencies. The data show distinctly different behavior in the two bands.

[3] The major finding is a high ratio of diurnal to semidiurnal internal tidal energy flux onto the shelf, approximately eleven to one, which exceeds the ratio of diurnal to semidiurnal barotropic tidal kinetic energy in the area by about a factor of three. The contrast between the two species is discussed in terms of volumetric vector forcing (spatially distributed forcing by the three-dimensional tidal current vector), nonlinearity, and the relationship between seafloor slope and internal wave ray slope.

[4] The measurements were collected during the Asian Seas International Acoustics Experiment (ASIAEX) of 2001. The South China Sea portion of ASIAEX was a joint endeavor of United States and Taiwan investigators. The site is roughly $450 \mathrm{~km}$ west of the Luzon Strait on the continental slope south of China (Figure 1). Separate from

\footnotetext{
${ }^{1}$ Woods Hole Oceanographic Institution, Woods Hole, Massachusetts, USA.

${ }^{2}$ Now at Applied Physics Laboratory, University of Washington, Seattle, Washington, USA.
}

Copyright 2008 by the American Geophysical Union. 0148-0227/08/2007JC004418\$09.00 diurnal internal tidal waves which appear to be generated in our work area, there are two types of internal waves incident on the site from deep water to the east: large transbasin nonlinear waves and internal tides, each presumably originating in the Luzon Strait area [Chang et al., 2006; Duda et al., 2004; Ramp et al., 2004]. One interpretetation of our observations is that some of the observed diurnal internal tidal energy on the shelf, and all of the semidiurnal, originated offshore to the east, with the remainder of the diurnal energy locally generated.

[5] The process of internal tide generation at this site is interesting for two reasons. First, barotropic tides at the site have four important constituents, two diurnal and two semidiurnal [Beardsley et al., 2004]. The diurnal band has a stronger fortnightly beat pattern than the semidiurnal, and the diurnal tides are also stronger. Next, the seafloor inclination is near critical for diurnal frequency internal waves over much of the instrumented area, and is subcritical for semidiurnal waves. Here, critical means having slope $s$ near that of the internal wave characteristics (raypaths, parallel to local group velocity), which is given by $c$, where $c^{2}=\left(\omega^{2}-f^{2}\right) /\left(N^{2}-\omega^{2}\right)$ and where $\omega$ is wave frequency, $N$ is buoyancy frequency, and $f$ is the Coriolis parameter. Subcritical means the seafloor lies at a lesser angle from the horizontal than the internal wave rays. The differing criticality of the two species allows a quantitative look at slope influence on internal tide generation (although other factors complicate this, such as internal tides propagating into the area interfering with local generation).

[6] High-fidelity simulation of internal tides requires highly accurate bathymetry, sometimes exceeding known accuracy, and also requires higher spatial resolution in the flow field gridding than is typically employed in regional models [Robertson, 2006]. Much better known are the 

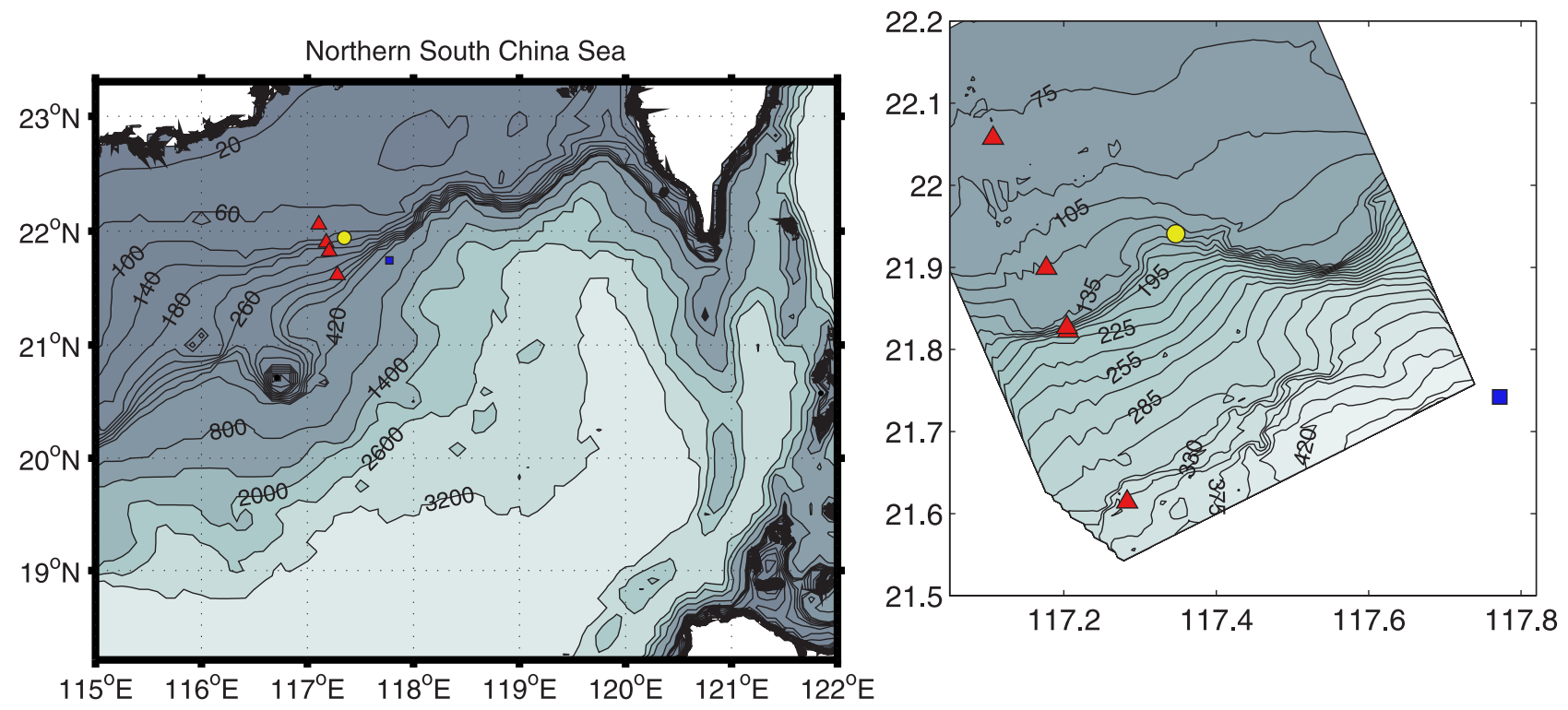

Figure 1. (left) A chart of the area. The locations of the four moorings whose data are used here to compute fluxes are shown with red symbols. Note that the mooring area, the east side of the Dongsha Plateau, has a seafloor depth gradient of lesser magnitude than a typical continental slope. To the right of the mooring line is a light (yellow) circle showing an additional mooring (T112, vertical thermometer string) and a dark (blue) square showing the position of the Chang et al. [2006] mooring IW3 cited in this work. (right) The area of the moorings is shown in detail, with the same moorings plotted. Measured bathymetry [Duda et al., 2004] is contoured. The main mooring line at the left is composed of, from north to south, S3, S4, S5, and S7. The Chang et al. [2006] IW3 mooring location is out of the range of the measured bathymetry on the right. Steps in the bathymetry can be seen. The region of uniform slope between S7 and S5 is inclined at $0.25^{\circ}$.

barotropic tidal surface displacements that force internal tides [Egbert et al., 1994]. It would be helpful to know aspects of seafloor topography and stratification that control the spatial distribution, mean level, and temporal variability of internal tide generation, so that precise bathymetry and increased grid resolution could be included in (at least) the most important areas. Despite what has been learned from modeling efforts at a few locations, the details of internal tide generation are not currently well enough known to allow parameterized internal tide related effects to be included with confidence in global ocean models and predictions. A separate issue is whether special focus must be given to areas of the seafloor with near-critical slope. A further complicating factor is that regional models of marginal seas may not have proper internal tides entering the model domain from generation sites across the basin, which may impact local generation.

[7] To investigate factors influencing internal tide generation in the field, this study explores the complexity and variability of the internal tide at the ASIAEX site, and looks at several potentially important factors. Behavior at the site contrasts with that of another low-latitude site on the Australian North-West Shelf studied by Holloway et al. [2001], which had dominantly semidiurnal surface and internal tides and different bathymetry. In particular, the ratio of baroclinic to barotropic wave energy flux in the dominant diurnal band at ASIAEX was higher, at 0.10, than the analogous ratio of 0.04 for the locally dominant semidiurnal band at the Australian site.

[8] Internal tides generated at the continental slope may propagate upslope, as do the waves examined here, or into deeper water (as examined by, e.g., Pingree and New [1991]). Baroclinic tides moving onto the shelf may be the primary source of energy for diapycnal mixing in those waters (when surface forcing is seasonally weak) at depths away from the surface mixed layer and the seafloor boundary layer (currents from barotropic tides, surface waves, and subtidal features induce mixing in the boundary layer). On the New England shelf, nonlinear internal waves, probably developed from slope-generated internal tides, were found to be the primary source of mid water column energy dissipation and mixing many tens of kilometers away from the shelfbreak [MacKinnon and Gregg, 2003]. On the other hand, internal tides at the Australian site were found to be generated and quickly dissipated (for the most part) in a confined area near the shelf edge, in water depths of $100-150 \mathrm{~m}$, weakening the stratification in that area [Holloway et al., 2001] via diapycnal mixing. Such a pronounced weakening of the stratification in the shelfbreak area was not observed in ASIAEX. A full energy budget, including barotropic tides, baroclinic tides, nonlinear waves developed from the tides, and dissipation, would be complex to measure and is certain to have geographic variability. Here we address only the forcing of baroclinic waves by barotropic waves, one portion of the energy budget problem.

[9] Section 2 describes the location of this study and the methods used. Section 3 shows the detailed internal tide energy flux results, and analyzes their relation to parameters such as bottom slope and local barotropic tides. Section 4 provides additional information about the internal tides in the area. Section 5 discusses the physics of internal tidal 

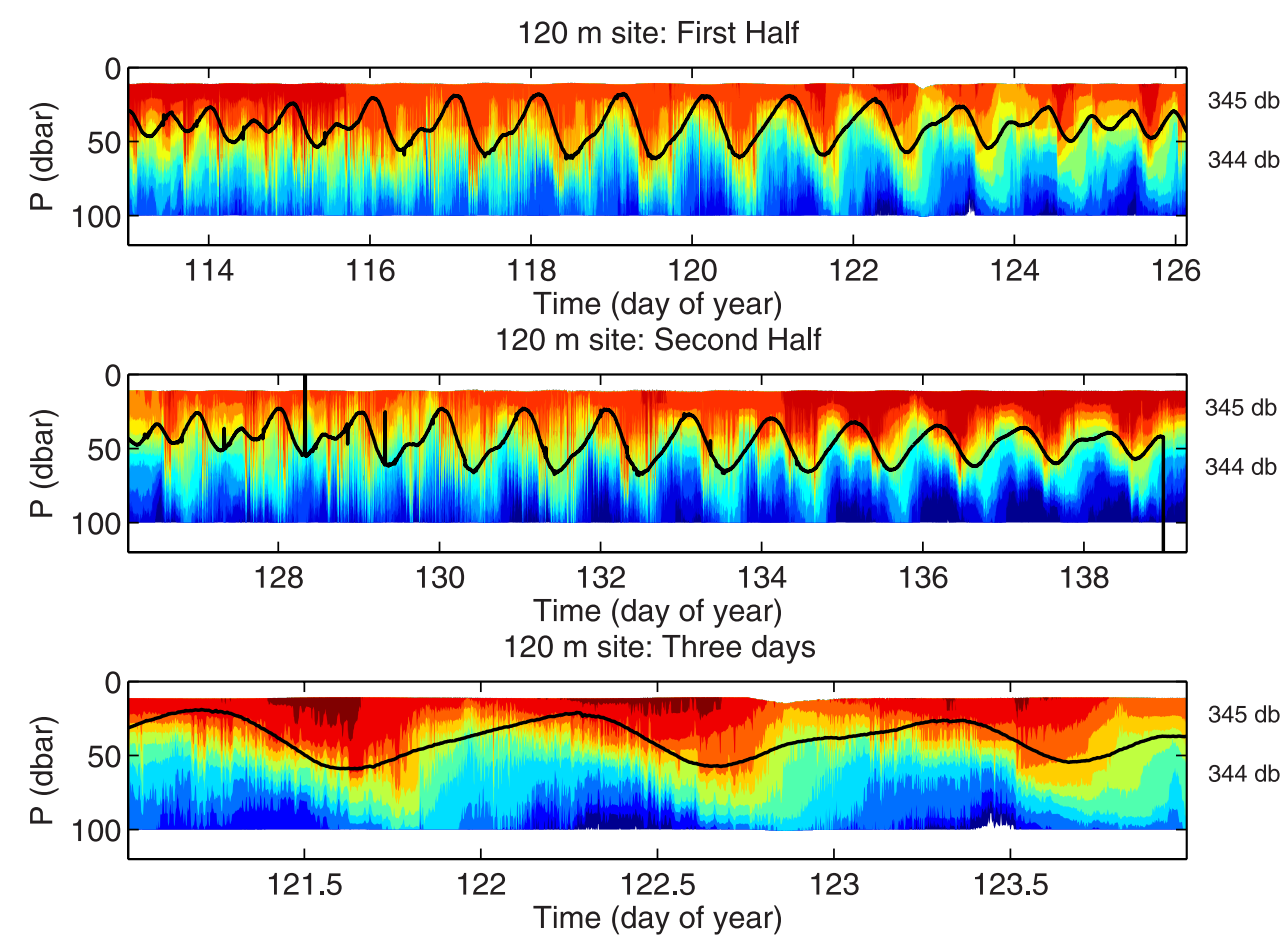

Figure 2. Isotherms at S4 are contoured. (top) The first half of the record. (middle) The second half of the record. (bottom) Three days from the first half with an expanded time scale. Contours are $17^{\circ}$ to $28^{\circ}$, interval $1^{\circ}$. Also shown (black line) is the pressure measured near the seafloor at S7, with pressure scales shown at the right.

generation and describes different properties in the two bands that may explain the stronger generation of diurnal internal tides. Section 6 summarizes the findings.

\section{Location and Methods}

[10] The data were collected during the April/May 2001 ASIAEX South China Sea study (Figure 1). The study area is northeast of the Dongsha Plateau, and is notable for the relatively shallow inclination of the continental slope, readily evident in the picture. The low-resolution version of the bathymetry shown at the left has a slope angle that is generally less than a degree, compared with $6^{\circ}$ or more around much of the basin.

\subsection{Moorings}

[11] Many moorings were deployed during this operation, with full description by Ramp et al. [2004]. The most heavily instrumented moorings were along a line with heading $340^{\circ}$ true, beginning at $71-\mathrm{m}$ water depth and extending southward off the shelf. From shallow to deep these are called S2 through S7. Here we analyze data from S3, S4, S5 and S7, the only moorings with records of current and temperature throughout the water column. The depths at these sites are 85, 120, 200, and $350 \mathrm{~m}$. Figure 1 shows the bathymetry of the area and the mooring locations in detail on the right. The bathymetric data are from a dedicated survey and other echo sounder data obtained during ASIAEX. The moorings are aligned roughly upslope.

[12] Instruments were placed on single moorings at S7, S4 (33 km from S7), and S3 (52 km from S7). Mooring site
S5 (24 km from S7) hosted two moorings; one with an ADCP and another with water column sensors.

[13] The data from S7 used here are current data from an upward looking RDI Broadband $300 \mathrm{kHz}$ acoustic Doppler current profiler (ADCP) at 100-m depth (data from 94.5 to $10.5 \mathrm{~m}, 4-\mathrm{m}$ bins), current data from Aanderra RCM8 current meters at 150-, 210-, and 310-m depth, and temperature data from various instruments at 9 depths (20, $40,60,80,142,152,201,261$, and $346 \mathrm{~m})$. The S5 site is near a small escarpment. Upward of the escarpment was mooring S5a at 184-m depth with an RDI narrowband $150 \mathrm{kHz}$ ADCP at $177 \mathrm{~m}$ (data from 165 to $37 \mathrm{~m}, 8-\mathrm{m}$ bins). Mooring S5 was located on the deep side of the escarpment, $0.5 \mathrm{~km}$ from S5a, where the water depth was $202 \mathrm{~m}$. S5 was equipped with instruments throughout the water column. The data used here are temperature recordings at 11-, 40-, 60-, 80-, 100-, 140-, 160-, and 180-m depth. From the S4 mooring at $120-\mathrm{m}$ depth, current data from an upward looking $300-\mathrm{kHz}$ broadband ADCP at $104 \mathrm{~m}$ depth are used (data from 98.5 to $14.5 \mathrm{~m}, 4-\mathrm{m}$ bins), plus temperature time series collected at 11-, 20-, 39-, 59-, $69-, 80-, 90-$, and 100-m depth. S3 data used here are from an upward facing broadband ADCP near the bottom (data from 74.5 to $10.5 \mathrm{~m}, 4-\mathrm{m}$ bins) and thermometers at 14-, 32-, 42-, and 72-m depth.

[14] Figure 2 shows temperature at S4 contoured versus depth and time for the duration of the experiment. The large dominantly diurnal internal tidal excursions in the center areas of Figures 2 (top) and 2 (middle) are easily picked out by eye. The pressure record at S7 is also dominantly 


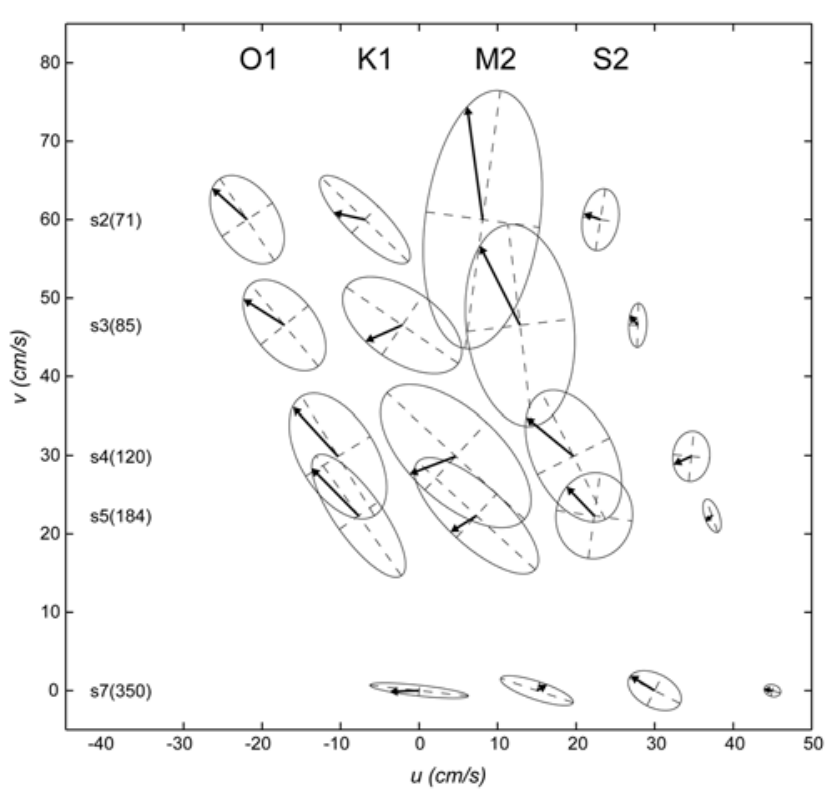

Figure 3. Barotropic tidal ellipses measured in the area. S3, S4, S5, and S7 are the sites for which we investigate baroclinic tidal energy flux. The semidiurnal and diurnal species have comparable current speeds to each other at those locations. Figure reprinted with permission from Beardsley et al. [2004] (C) 2004 IEEE).

diurnal, and has a high degree of correlation with isotherm displacements.

\subsection{Stratification and Seafloor Inclination}

[15] The seafloor in the area has an interesting terraced geometry at horizontal scale of tens of kilometers. A detailed image made with a towed chirp sonar is given by Schock [2004]. Seaward of the shelfbreak, which is between S4 and S5, there are lengthy regions of relatively uniform slope of about $0.25^{\circ}$ separated by escarpments that are tens of meters high and that are poorly measured by echo sounders. The escarpments are supercritical for all tidal frequencies. The slopes along the mooring line between the escarpments are quite uniform if short-scale deviations of a few hundred meters wavelength or less are averaged over.

[16] The observed slope of $0.25^{\circ}$ is subcritical in the diurnal band for buoyancy frequency $N<5.2 \mathrm{cph}$. For larger $N$ the seafloor is supercritical. This slope is subcritical in the semidiurnal band for $N<17 \mathrm{cph}$. During ASIAEX the maximum $N$ was typically 10 to $12 \mathrm{cph}$, at depths between 50 and $70 \mathrm{~m}$, so the seafloor slope can be considered subcritical for semidiurnal frequencies in all of the gently sloping regions. In a later section, the implications of seafloor slope in this area on the matching of barotropic tide water parcel orbits and theoretical internal wave orbits, and presumably on internal tide generation, is presented.

\subsection{Tidal and Internal Tidal Flow}

[17] The barotropic tidal current field has been determined using harmonic analysis of ADCP records at five ASIAEX sites, including our four mooring positions. Beardsley et al. [2004] describe the barotropic tidal constituent amplitudes, phases, current ellipses, and energy fluxes.
In the procedure, velocities of uppermost and lowermost bins were extended to the surface and bottom before vertical averaging and harmonic analysis of the mean time series. Harmonic analysis is performed using a least squares method [Pawlowicz et al., 2002]. All resolved constituents were allowed in the analysis procedure, including overtones (M3, M4 etc.). Figure 3 shows current ellipses for four constituents (two diurnal and two semidiurnal) at the five locations.

[18] Figure 4 (top) shows eastward current at S5. Also shown are a low-pass-filtered subtidal component (periods greater than 33 hours) and a bandpass filtered component containing tidal frequency motions. Figure 4 (middle) shows eastward $U_{x}$ and northward $U_{y}$ tidal currents. Figure 4 (bottom) shows $U_{x}$ broken down into diurnal and semidiurnal components using a digital filter. The semidiurnal current is relatively steady (small fortnightly variation) because M2 energy greatly exceeds $\mathrm{S} 2$, while the diurnal has a strong fortnightly variation. Time $(t)$ is given in day of year 2001, where $1<t<2$ refers to 1 January and $120<t<121$ refers to 30 April.

[19] Tidal analyses of isotherm displacements and baroclinic currents at S4, S5 and S7 appear in prior work [Duda et al., 2004]. In this paper the baroclinic tide energy and energy flux are examined in greater detail, but first the prior results will be reviewed.

[20] The diurnal internal tides were found to have downward phase propagation at S5 and S4, consistent with upward raypaths. This result stems from harmonic analysis of residual currents after removal of barotropic flow, and from observation of isopycnal displacements, which reach maxima later at deep positions compared with shallow positions (Figure 2). Somewhat inconsistently with this, the currents at S4 and S5 had minima near the center of the water column, suggesting that the energy had organized itself into a mode one-like form. The diurnal internal tide phase velocity was found to be near $1.2 \mathrm{~m} / \mathrm{s}$, and the wavelength $110 \mathrm{~km}$. The semidiurnal internal tide phase speed was $0.7 \mathrm{~m} / \mathrm{s}$, wavelength $30 \mathrm{~km}$. Ellipse inclinations suggested that the waves were traveling at a heading of about $325^{\circ}$, roughly up the mooring line.

[21] At S4 the strongest diurnal internal tidal surface bores were observed to spawn nonlinear high-frequency internal waves at their leading edges. Somewhat differently from the behavior at the site of Holloway et al. [2001], where internal tide energy was not found to penetrate onto the shelf but was instead found to be formed and dissipated within a 30-km-wide region where the depth ranged from 150 to $100 \mathrm{~m}$ (based in part on variations of $N(z)$ attributed to dissipation), the diurnal internal tides illustrated in Figure 2 propagate at least $10 \mathrm{~km}$ inshore of S3 with great strength (based on additional mooring measurements not shown here), having displacements exceeding $20 \mathrm{~m}$ in water of $75-\mathrm{m}$ depth. This would be approximately $40 \mathrm{~km}$ inshore of S5.

\subsection{Data Analysis Techniques}

[22] The velocity and temperature time series data, all with a native sampling rate of one per minute or higher, are used to compute available potential energy, kinetic energy, and baroclinic wave energy flux in the diurnal and semidiurnal frequency bands. 

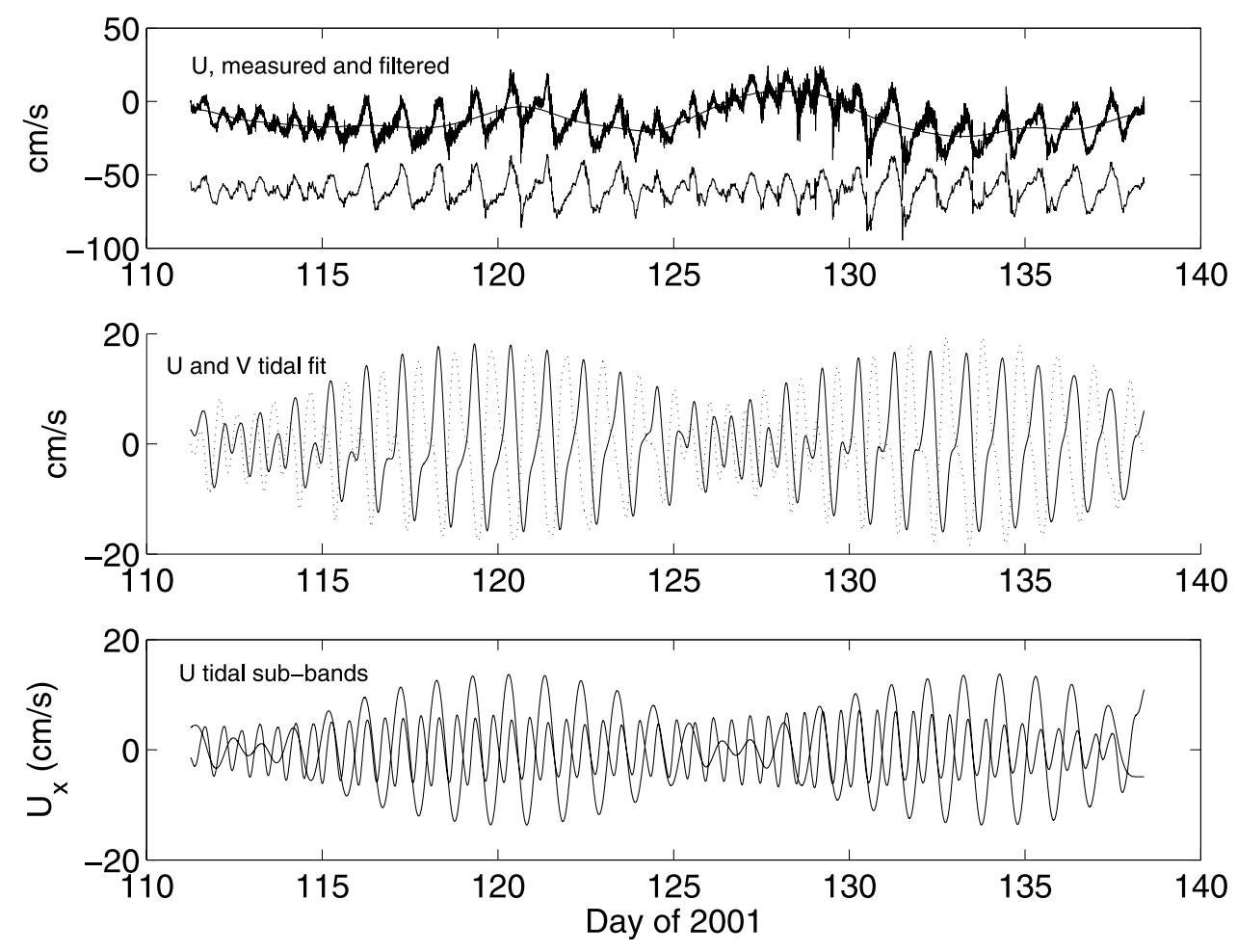

Figure 4. (top) Depth-mean eastward currents at S5 are shown. The depth-mean time series is shown at the top. Overlain on that time series is a subtidal band low-pass-filtered version. Offset below is a bandfiltered version that includes tidal periods. (middle) Fitted east and north (dash) tidal currents $\left(U_{x}\right.$ and $\left.U_{y}\right)$ are shown [from Beardsley et al., 2004]. (bottom) Semidiurnal and diurnal portions of the tidal fit eastward current $U_{x}$ are shown. A single low-pass-filtering operation was used to separate the two.

[23] The depth-integrated fluxes for a given frequency band are given by

$$
\mathbf{F}=\int_{-h}^{0} \mathbf{F}_{\mathbf{A}}(z) d z=\int_{-h}^{0}\left\langle p^{\prime}(z) \mathbf{u}_{\mathbf{i}}(z)\right\rangle d z
$$

which has the units watts per meter. The water depth is $h$, and $p^{\prime}$ and $\mathbf{u}_{\mathbf{i}}$ are estimates of baroclinic pressure and velocity fluctuations resulting from the signal processing described below. The brackets indicate time average. The flux of a sinusoidal wave would be correctly computed by averaging over an integer number of wave periods. Here, the product $p^{\prime} \mathbf{u}_{\mathbf{i}}$ is computed using demodulated signals in each frequency band, relaxing the requirement to average over an integer number of wave periods.

[24] The velocity data are processed using some of the methods described by Nash et al. [2004], Rainville and Pinkel [2006], and Alford and Zhao [2007]. After subtraction of barotropic tidal velocity time series described in section 2.3, time series of baroclinic velocity $\mathbf{u}_{\mathbf{i}}$ and temperature are band-passed around the dominant diurnal $(\mathrm{K} 1=$ 1.003 cycle per day (cpd)) and semidiurnal $(\mathrm{M} 2=$ $1.932 \mathrm{cpd}$ ) tidal frequencies. Fourth-order Butterworth filters are used, with passbandwidth of $0.4 \mathrm{cpd}$. This relatively wide-band filtering separates the two bands of interests while capturing their time variability (e.g., the fortnightly cycle). Note that inertial waves $(\omega=\mathrm{f} \sim$ $0.75 \mathrm{cpd}$ ) are excluded from the diurnal band.
[25] The top-to-bottom displacement profile $\eta(z)$, from which the baroclinic pressure profile $p^{\prime}(z)$ can be computed, is estimated from the discrete vertical temperature measurements by fitting vertical modes to isopycnal displacement estimates. Isopycnal displacements at the depths of the each temperature sensor $\left(z_{i}\right)$ are obtained from band-passed temperature time series $\left(T_{b p}\left(t, z_{i}\right)\right)$ by assuming local vertical advection of the background temperature field $\bar{T}$ via

$$
\eta\left(t, z_{i}\right)=T_{b p}\left(t, z_{i}\right)[d \bar{T} / d z]^{-1}
$$

In this data set the first three vertical dynamical modes, calculated using the averaged observed buoyancy frequency profile at each mooring site, account for practically all of the variance observed in the displacement time series. The topto-bottom displacement field is obtained by summing all the modes. Using the hydrostatic equation, the baroclinic pressure field $p^{\prime}(t, z)$ is then computed, with the choice of the integration constant such that the vertical integral of $p^{\prime}(t, z)$ is equal to zero.

[26] The band-pass filtering (centered at a frequency $\omega$ ) of the vertical displacement is done via complex demodulation, leading to an estimate of the form $\eta\left(t, z_{i}\right)=$ $\alpha\left(t, z_{i}\right) \cos \left(\omega t+\phi\left(t, z_{i}\right)\right)$, where $\alpha$ and $\phi$ are the amplitude and phase of the signal, slowly varying on the timescale of the filter bandwidth, here 2.5 days, at the depth $z_{i}$ of the measurement. These amplitudes and phases at discrete depths are then used to estimate the continuous vertical structure by fitting three vertical modes, resulting in slowly 

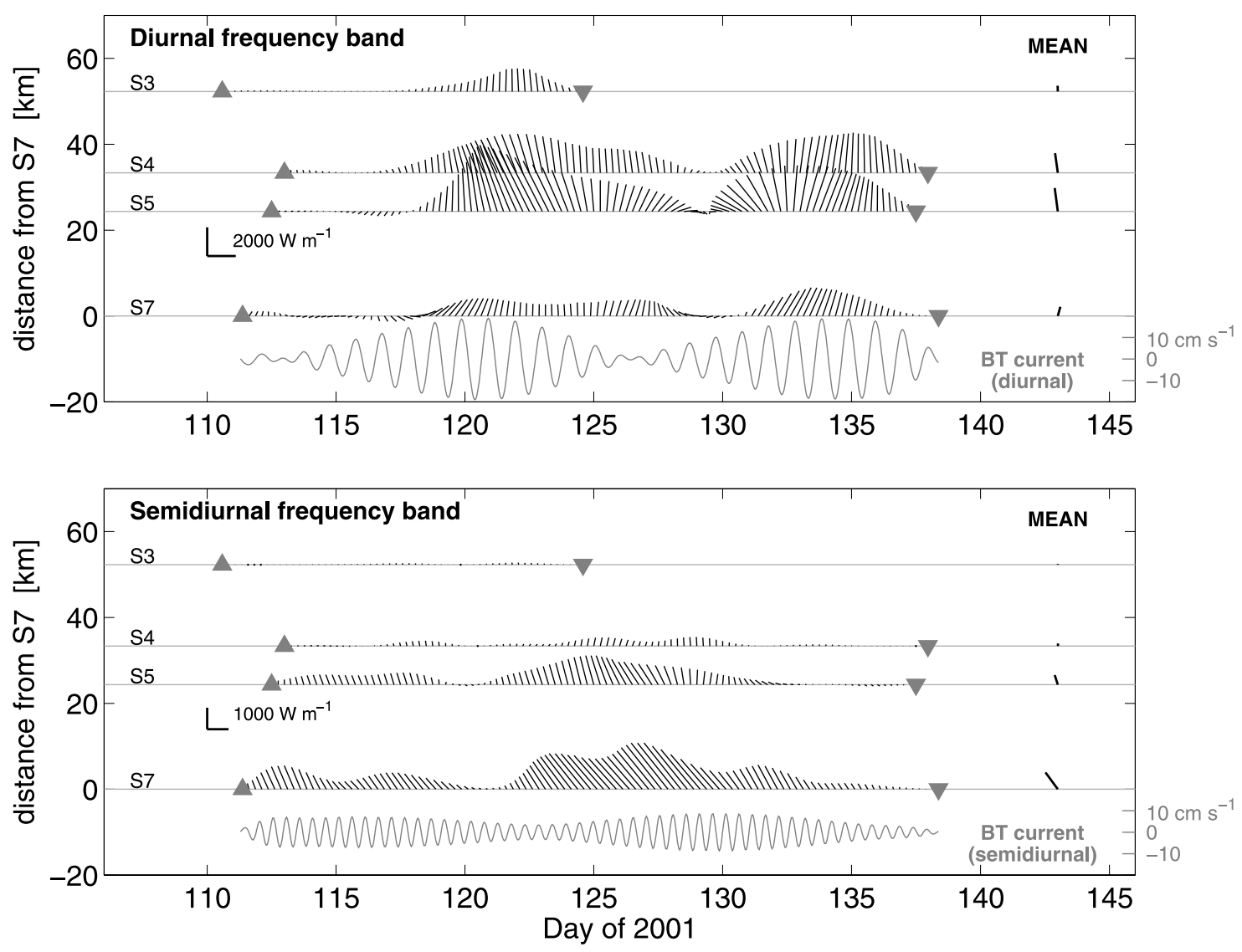

Figure 5. Time series of energy flux $\mathbf{F}$ for (top) diurnal and (bottom) semidiurnal species, rotated so that a vertical arrow corresponds to upslope flux in the direction $340^{\circ}$ (i.e., up the mooring line). The up and down triangles indicate the start and end, respectively, of each time series. The cross-shelf diurnal and semidiurnal barotropic currents at S5 are plotted.

varying amplitude $A_{m}(t)$ and phase $\Phi_{m}(t)$ time series describing the entire time-depth domain $\eta(t, z)=$ $\sum_{m} A_{m}(t) \Psi_{m}(z) \cos (\omega t+\Phi(t))$ where $\Psi_{m}$ are the three mode shapes $(m=1$ to 3$)$. A similar mode decomposition and full depth synthesis is performed on the filtered baroclinic velocity in each band. The depth-integrated energies and product $p^{\prime} \mathbf{u}_{\mathbf{i}}$ (and thus $\mathbf{F}$ ) are computed in each frequency band using the $A_{m}(t)$ and phase $\Phi_{m}(t)$. As a result, the effective temporal averaging is over the timescale of the bandwidth, 2.5 days (five periods for semidiurnal, less than three for diurnal).

[27] Variation of filter bandwidths and demodulation frequencies provides a method to estimate errors and confidence intervals. Two alternative analysis methods to that described above gave temporal mean flux component estimates that differed from the numbers published here by $2 \%$ to $14 \%$.

\section{Internal Tide Energy Flux}

[28] Figure 5 shows time series of baroclinic energy flux vectors $\mathbf{F}$ at each site. These are shown for the diurnal and semidiurnal bands. The coordinates are rotated so that flux drawn in the positive $y$ direction is at heading $340^{\circ}$. At the right of the time series are the mean values for each site, calculated over either 14 or 28 days (approximately). Table 1 gives flux magnitudes and directions (with respect to true north). Figure 6 shows time mean $\mathbf{F}_{\mathbf{A}}$ (flux per unit area) versus depth for the two species at the four moorings. Also drawn are internal wave characteristics calculated using a smooth estimate of the mean stratification measured by the moorings. The diurnal frequency characteristics are roughly parallel to the seafloor.

[29] The mean and peak flux values are larger in the diurnal band than the semidiurnal band (note the scale difference in Figure 5). The fluxes are directed toward the coast and are somewhat in alignment with the mooring line.

Table 1. Parameters for Tidal Species at Four Sites ${ }^{\mathrm{a}}$

\begin{tabular}{lcccc}
\hline Site/Species & BC Flux & BT Flux & $\begin{array}{c}\text { BT Ellipse } \\
\text { Inclination }\end{array}$ & $\begin{array}{c}\left\langle\vec{U}^{2}\right\rangle, \\
\mathrm{cm}^{2} / \mathrm{s}^{2}\end{array}$ \\
\hline S3 diurnal & $0.41 @ 338^{\circ}$ & $8.9 @ 58^{\circ}$ & O1-129 $/ \mathrm{K} 1-146^{\circ}$ & 79 \\
S4 diurnal & $1.39 @ 332^{\circ}$ & $13 @ 70^{\circ}$ & $\mathrm{O} 1-121^{\circ} / \mathrm{K} 1-137^{\circ}$ & 142 \\
S5 diurnal & $1.66 @ 332^{\circ}$ & $14 @ 57^{\circ}$ & $\mathrm{O} 1-125^{\circ} / \mathrm{K} 1-137^{\circ}$ & 112 \\
S7 diurnal & $0.68 @ 355^{\circ}$ & $17 @ 271^{\circ}$ & $\mathrm{O} 1-174^{\circ} / \mathrm{K} 1-162^{\circ}$ & 36 \\
S3 semidiurnal & $0.03 @ 339^{\circ}$ & $6.6 @ 346^{\circ}$ & $\mathrm{M} 2-97^{\circ} / \mathrm{S} 2-88^{\circ}$ & 146 \\
S4 semidiurnal & $0.12 @ 348^{\circ}$ & $5.9 @ 318^{\circ}$ & $\mathrm{M} 2-117^{\circ} / \mathrm{S} 2-84^{\circ}$ & 69 \\
S5 semidiurnal & $0.46 @ 322^{\circ}$ & $6.2 @ 328^{\circ}$ & $\mathrm{M} 2-82^{\circ} / \mathrm{S} 2-109^{\circ}$ & 37 \\
S7 semidiurnal & $0.98 @ 304^{\circ}$ & $9.0 @ 303^{\circ}$ & $\mathrm{M} 2-153^{\circ} / \mathrm{S} 2-164^{\circ}$ & 10.7 \\
\hline
\end{tabular}

${ }^{\mathrm{a}}$ The first data column contains mean energy flux of baroclinic (BC) tides. The remaining columns contain barotropic (BT) tide information. Flux quantities are magnitude $(\mathrm{kW} / \mathrm{m})$ and direction (True). Inclination is measured in degrees anticlockwise from east $\left(90^{\circ}\right.$ true). 

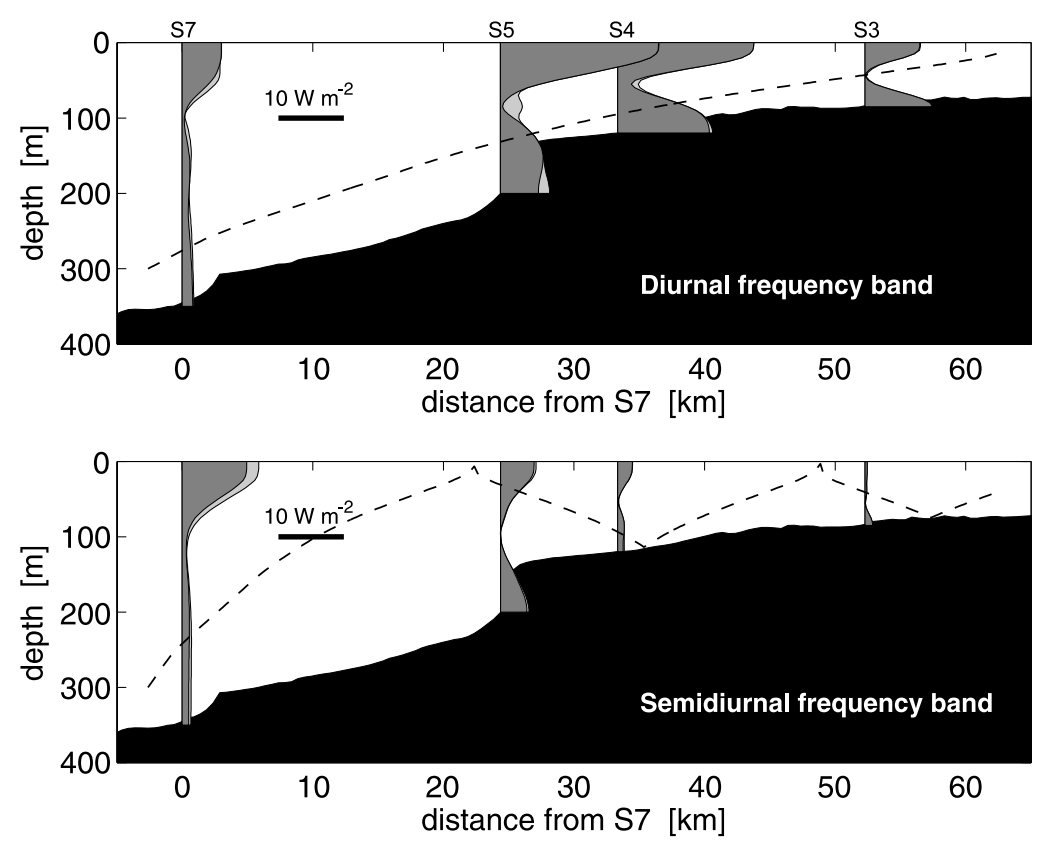

Figure 6. The temporal average of $\mathbf{F}_{\mathbf{A}}$ as a function of depth at each site: (top) diurnal band and (bottom) semidiurnal band. Bathymetry is indicated in black. Light gray is the magnitude of the flux, and dark gray (often nearly equal to light grey) is the component directed upslope. One characteristic entering the domain is drawn for each band.

The mean and instantaneous diurnal band $\mathbf{F}$ are greater in magnitude at the shallow sites (S5 and S4) than at S7, and they are directed away from S7 (for the most part), which strongly suggests generation along the mooring line, particularly the region between S7 and S5. Note that lower diurnal flux at S4 than S5 may be due in part to the strong tidal overtones of the nonlinear internal tide attenuating the output of the flux analysis because first overtones of diurnal oscillations will be in the semidiurnal band (the internal tides at S4 show a strong sawtooth pattern, Figure 2). Overall, the evidence suggests a source of diurnal baroclinic energy at depths between those of S7 and S5. Profiles of energy flux (Figure 6) show enhancement both near the surface and near the bottom. The spatial resolution of the mooring array is not sufficient to discuss the generation process in terms of beams, or to rule out horizontal convergence of energy flux.

[30] In contrast, the semidiurnal flux is greatest at S7, where a strong presence of internal tidal energy originating in the Luzon Strait is expected [Chang et al., 2006]. It is impossible to tell from this analysis whether this energy is indeed from the Luzon Stait, or from a less distant source.

[31] Semidiurnal energy flux monotonically decreases moving up the line into shallower water. The observation that all four time series of flux have similar temporal form suggests that local generation is not occurring, and that energy is arriving from the east. Either the energy dissipates as the waves propagate up the slope and onto the shelf, incident energy decreases to the north, or there are horizontal refraction (divergence) effects. The direction of the semidiurnal energy flux is not well-aligned with the mooring line: the direction of S7 flux is not toward S5, and so on. It is unlikely that this is a two-dimensional problem aligned with our mooring line, with no along-shelf dependence. Regardless of the ambiguous meaning of the decreasing flux along the line, there is no evidence of enhancement of semidiurnal internal tide flux via local generation.

[32] Under the assumption that none of the semidiurnal internal tides are locally generated, which cannot be strictly ruled out in light of our observation geometry (which prevents us from calculating a complete energy budget in the horizontal), the implied infinite ratio of locally generated diurnal $\mathbf{F}$ to locally generated semidiurnal $\mathbf{F}$ far exceeds the ratio of barotropic tide diurnal to semidiurnal kinetic energy (Table 1). The excess diurnal internal tide flux at S4 over S7 is $0.7 \mathrm{~kW} / \mathrm{m}$, far greater than the semidiurnal internal tide flux at $\mathrm{S} 4$ of $0.12 \mathrm{~kW} / \mathrm{m}$. If the $\mathrm{S} 4$ semidiurnal internal tide was entirely locally generated, then the ratio of excess diurnal to semidiurnal baroclinic fluxes at $\mathrm{S} 4$ is almost $6: 1$, whereas ratio of barotropic kinetic energies at S7 decomposed into the two species (not tide line constituents) is about 3.4:1. (The barotropic energy flux depends on the phase of the currents and the surface displacement, and does not necessarily reflect the energy available locally for conversion to internal tides.) The critical nature of the slope is a possible explanation for the large ratio of mean diurnal to semidiurnal internal tides, as are elliptical forcing trajectories. These two effects are examined in sections to follow.

[33] The internal tide energy fluxes (Figure 5) are less regular over time than the forcing tidal currents in each species (Figure 4 (bottom)), suggesting that factors other than forcing current amplitudes, parsed by species, particle trajectories, and the seafloor slope play a role. These can be variable stratification (not seen in our records), and variable subtidal currents (which we do not examine).

[34] The S7 and S5 internal tide energy fluxes differ from 20-day mean fluxes reported for an ADCP-instrumented site 
Table 2. Mean Energy Levels of Internal Tide Species by Site ${ }^{\mathrm{a}}$

\begin{tabular}{lccc}
\hline Site/Species & KE & APE & Total \\
\hline S3 diurnal & 680 & 290 & 970 \\
S4 diurnal & 2600 & 500 & 3100 \\
S5 diurnal & 1800 & 1000 & 2800 \\
S7 diurnal & 1540 & 480 & 2020 \\
S3 semidiurnal & 97 & 21 & 120 \\
S4 semidiurnal & 350 & 60 & 410 \\
S5 semidiurnal & 600 & 220 & 820 \\
S7 semidiurnal & 670 & 260 & 930 \\
\hline
\end{tabular}

${ }^{\mathrm{a}}$ Kinetic energy (KE), available potential energy (APE), and the sum of the two are shown. Units are $\mathrm{J} / \mathrm{m}^{2}$.

to east of our mooring line at $21^{\circ} 44.53^{\prime} \mathrm{N}, 117^{\circ} 46.31 \mathrm{E}$ where the depth is $468 \mathrm{~m}$. At that site, Chang et al. [2006] report semidiurnal flux of $4.1 \mathrm{~kW} / \mathrm{m}$ toward $278^{\circ} \mathrm{T}$ and diurnal flux of $4.2 \mathrm{~kW} / \mathrm{m}$ toward $345^{\circ} \mathrm{T}$. The higher fluxes of Chang et al. [2006] are computed, via good methods, using the formula flux equal to group velocity times energy density, and using data from the upper half of the water column. It is possible but not likely that their use of normal mode analysis to compute the full water column integral may lead to errors. They also used only a 20-day sample, so there may be a biased average of the mean of a fortnightly varying internal tide. Internal tides passing by their mooring in multiple directions might lead to erroneous fluxes using their group velocity method. On the other hand, it is possible that our observations are from a time period of reduced internal tide compared to their time period (April 2000). Direct comparison of their time series and our time series would be needed to verify and explain the discrepancy. The Chang et al. [2006] diurnal energy density is 0.9 times ours at S7 (Table 2), while their semidiurnal energy density is 2.6 times ours. Group velocities inferred using our internal tide energies (Table 2) and fluxes (Table 1) fluctuate by about factor of 1.7 (diurnal) and 4.0 (semidiurnal), illustrating limitations to the group velocity approach, and possibly also disclosing suitable confidence intervals for our energy and flux computations.

\section{Other Internal Tide Parameters}

[35] Some basic properties of the internal tidal displacement and current fields were presented in section 2.3. Quantitative details of the internal tide presented by Duda et al. [2004] are repeated here to put the flux observations into context.

[36] Harmonic tidal analysis was performed on time series of isotherm displacement and baroclinic velocity (barotropic component removed) from S4, S5, and S7. The fitted current ellipses show minor-to-major axis ratios hovering near $f / \omega$ for both species, with some jitter in the results. Constituent flow having this ratio suggests a single plane wave following internal wave kinematics, with the ellipse inclination giving the direction of propagation. These inclinations are west of north at most depths for all $\mathrm{O} 1, \mathrm{~K} 1$, M2 and S2 constituents, roughly running up the line of moorings $\left(340^{\circ}\right)$. The phase relationships of currents at S5 and S4 yield (upper bound) wavelengths $2 \pi / k_{h}$ of order $100 \mathrm{~km}$ for diurnal constituents, and $30 \mathrm{~km}$ for semidiurnal. Resulting phase velocities along the line (and upper bound phase speeds $c_{p}=\omega / k_{h}$ ) are $1.1 \mathrm{~m} / \mathrm{s}$ for diurnal constituents, $0.7 \mathrm{~m} / \mathrm{s}$ for semidiurnal.
[37] The mean kinetic, potential, and total energies of the internal tides in the two bands show behavior similar to the energy flux. The semidiurnal energy decreases shoreward (Table 2) and the diurnal increases shoreward (except for S3). The similar (but not identical) behaviors of flux and energy are expected because flux equals energy times group velocity, and group velocity is slowly varying.

[38] At S4, the isotherm displacements show phase behavior with depth consistent with downward phase velocity and upward group velocity and wave number for the dominant diurnal signal (Figure 2). This trend is also evident in the harmonically fitted internal tide time series, and in the depth-dependent tidal ellipse phases derived from the ADCP records. However, the phase relationships between the tidally fitted currents and displacements do not show the expected relationships. The displacements lag the horizontal velocities in the direction of tide propagation, as expected, but the phases depart from the expected quadrature. This may be due to nonlinearity of the internal tides, evident in sawtooth-type displacement time series indicative of higher harmonics (Figure 2).

[39] At S4, the strongest diurnal surface flood internal tides have a bore-like behavior at the surface, and they show a rapid rotation of current, with flow starting out toward the left of $340^{\circ}$, veering to that heading, then continuing to veer to the right as the surface isotherms plunge with the passing of the bore. The distorted waveforms with high steepness, and possible strong modulation of the internal tides, only allow approximately $50 \%$ of the baroclinic current variance to be fit by tides, versus 80 to $93 \%$ of barotropic current variance that fits tides (auxiliary material). ${ }^{1}$ Finally, internal tide displacements at two locations on the shelf are very similar (Figure 7), providing evidence that horizontal refraction is not the cause of strong diurnal internal tides at S4 and S5.

\section{Volumetric Vector Forcing of Internal Tides}

[40] The generation of internal waves is often considered to be a linear process. However, most modern simulations of internal tides utilize nonlinear primitive equation models and do not provide evidence of this. One such study shows good agreement between modeled and observed internal tides at the Hawaiian ridge, where the M2 tide strongly dominates (a different situation than here), with some evidence that the M2 and S2 internal tides behave linearly [Holloway and Merrifield, 2003]. However, very large overturns in areas of generation indicate that many nonlinear processes occur along the ridge [Levine and Boyd, 2006]. Unlike Hawaii, diurnal and semidiurnal species are forced at the same order of magnitude at the ASIAEX site. The fact that the ASIAEX baroclinic response does not match the barotropic forcing (Figure 5) might be an indication that the process is not linear, although other factors may influence response differently in the two bands, such as stratification, background currents and shear, and interfering incident internal tides from Luzon Stait of other places.

[41] To explore linearity and the effect of periodic flow orientation on wave generation, we investigate mechanisms

\footnotetext{
${ }^{1}$ Auxiliary materials are available in the HTML. doi:10.1029/ 2007JC004418.
} 

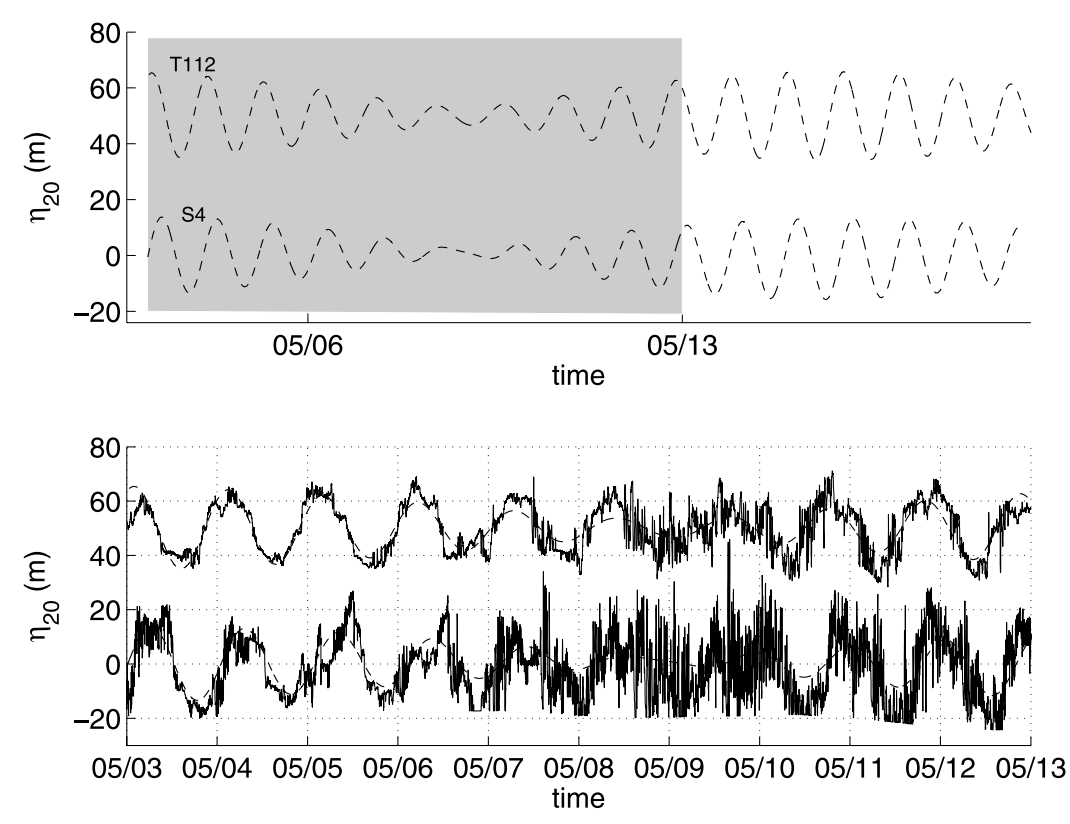

Figure 7. Isotherm displacement at two locations, T112 and S4, are shown. (top) Harmonically fitted tidal displacements of the $20^{\circ} \mathrm{C}$ isotherm are shown for the entire observation interval. The T112 displacements are offset $50 \mathrm{~m}$ in the plot. (bottom) The portions of the curves in the shaded portion of Figure 7 (top) are repeated (10 days), along with the isotherm displacements entered into the fit, interpolated from vertically spaced thermometers.

by which barotropic tide velocity directly enters into baroclinic wave forcing. This velocity does not explicitly appear in linearized forcing equations, but would appear with the addition of nonlinear terms.

[42] The term volumetric vector forcing can be applied to the hypothesized role of the barotropic flow process because the velocity vector is involved directly in the forcing process, and the spatial extent (volume) of coherent forcing regions must also play a role.

\subsection{Observed Internal Tides and Forcing Trajectories}

[43] The linearized equation most frequently used to diagnose internal tide generation is given by Baines [1982] and papers cited therein. The effect of current, and thus current trajectory, enters only through its effect in creating a density perturbation via vertical motions induced by barotropic waves traveling over a sloped seafloor. Similarity between forcing particle trajectory and internal wave orbits does not enter in the generation process. For the purposes of this discussion, one might assume that if the particle trajectory forced by the barotropic current were similar to that of an internal wave at the prescribed frequency, a free wave would be effectively generated. This situation is not transparently treated by the linearized equation.

[44] In this data set, it is noteworthy that barotropic tidal particle trajectories are favorable for diurnal internal tide generation during brief periods, and are favorable for semidiurnal internal tide generation during other brief periods. (By favorable, it is meant that the trajectories make single undisturbed anticyclonic loops during an internal wave period, so that they fit trajectories of freely propagating internal waves, including trajectory inclination parallel with a characteristic.) Figure 8 illustrates this by showing extracted sections of S5 horizontal tidal current, and by showing S5 particle trajectories computed by integrating the current. Presumably the flow is deflected to be parallel with the seafloor, with deflection trending to zero at the surface. For the illustration, the duration of the experiment is divided into ten equal length sequential time windows. Diurnal periods of 25 hours length at the center of each window are then selected. The $U_{y}$ and $U_{x}$ currents for each selection are plotted in Figure 8 (middle), along with the trajectory (top). In Figure 8 (bottom), the trajectory for the first 12.42 hours of the selected interval (an M2 semidiurnal time period) is also plotted. The circles show the beginning of each trajectory. Five of the diurnal period trajectories, indicated with bold box outline, are elliptical single loops that resemble particle trajectories satisfying the internal wave equations. Three single-loop semidiurnal period trajectories resembling internal wave trajectories are also indicated with bold box outline. Thus the outlined time periods are expected to have strong internal tide response for the respective species.

[45] Figure 9 shows flux vectors at ten equally spaced times that match up with the time windows of Figure 8. The times of loop-like particle trajectories (marked by bold boxes in Figure 8) show strong internal tidal response of the respective species. At the lower edge, the arrows indicate the possibility that the strongest semidiurnal flux may have passed to the south of the mooring line, which would agree with Chang et al. [2006]. Only a weak suggestion of local semidiurnal baroclinic response at S5 can be seen, in the sixth and seventh intervals, with refraction of the apparent transbasin internal tide seen at $\mathrm{S} 7$ to a more northerly direction at S5 and S4 also being a possibility. This contrasts with the local diurnal generation 

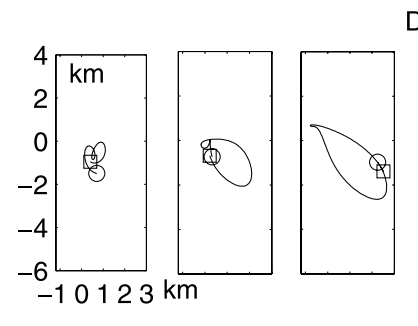

Diurnal time interval trajectories
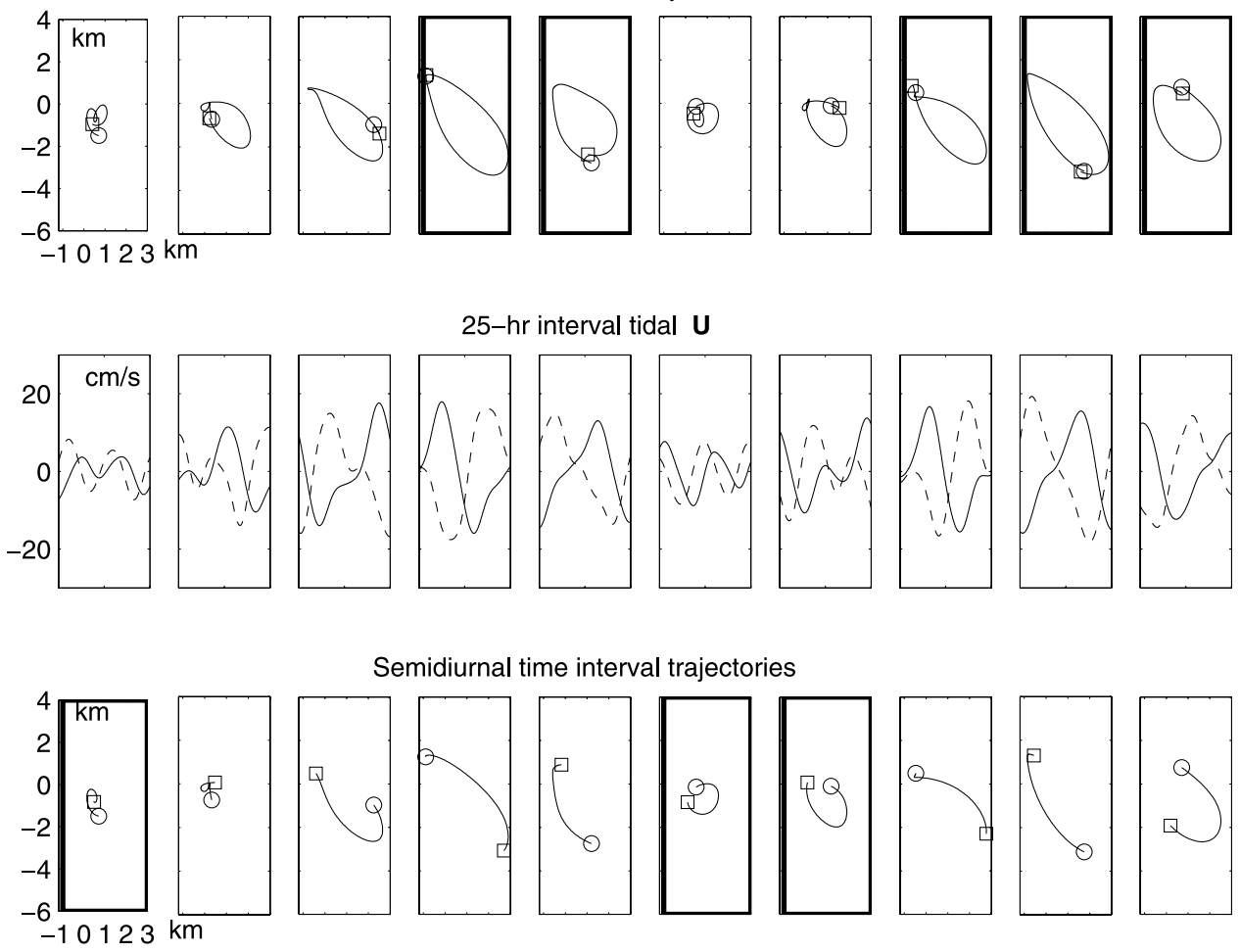

Semidiurnal time interval trajectories

Figure 8. Particle trajectories synthesized from the S5 tidal series $U_{x}$ and $U_{y}$ are shown for 10 sequential intervals encompassing the observations, with time moving from left to right. Each interval is 65-h long. (top) Trajectories during 25-h sections of the intervals, showing behavior ranging from single loops to deformed dual loops. Trajectory beginnings are marked with circles, ends are marked with squares. (bottom) Trajectories from 12.42-h long sections. For each species, times with simple loop trajectories expected to force internal tides are indicated with bold boxes. (middle) The current time series for the 25 -h subsections of the $65-\mathrm{h}$ intervals, from which the trajectories are computed.

implied by the evidently divergent energy fluxes shown in the central (diurnal) panels.

[46] The time variability of the local diurnal baroclinic response, nor the differences between diurnal and semidiurnal response, cannot be explained by changes in the stratification because the density profiles at each mooring did not change appreciably over the course of the experiment. Subtidal current variations (Figure 4 (top)) are not negligible, but these alone cannot explain both the semidiurnal and diurnal internal tide variability because the semidiurnal and diurnal internal tide flux variations are out of phase with each other.

[47] There is a primarily fortnightly temporal pattern of diurnal barotropic current but the pattern does not closely match the temporal pattern of the diurnal internal tides (Figure 5). The semidiurnal barotropic tides are quite regular (Figure 5) and also do not match the temporal pattern of semidiurnal internal tides.

[48] In particular, Figure 5 shows that diurnal $\mathbf{F}$ is relatively small before day 120 even though diurnal barotropic currents are large. These are times with large semidiurnal barotropic currents. Large diurnal $\mathbf{F}$ appear when the semidiurnal barotropic currents wane at day 120. In similar fashion, the largest semidiurnal $\mathbf{F}$ are observed at times with small diurnal barotropic currents (even though it has been pointed out that these data do not strongly identify local generation of semidiurnal internal tides, with a strong possibility that all the semidiurnal $\mathbf{F}$ has origin east of the mooring zone). These two timing behaviors support the hypothesis that near-elliptical barotropic particle trajectories, properly phased with vertical displacements, are more effective internal tide producers than the displacements alone.

\subsection{Forcing Equation}

[49] Here, a baroclinic response equation similar to the Baines equation is introduced and examined. The new equation has additional velocity vector (particle orbit) terms. The equation stems from the nonlinear equations for rotating stratified inviscid flow. These equations are now routinely solved to determine joint solutions for baroclinic and barotropic tidal waves, so there is no attempt to solve the derived equation. The derived equation may be most useful for diagnostics.

[50] Writing velocity as the sum of barotropic tide and baroclinic tide components $\mathbf{u}=\mathbf{U}+\mathbf{u}_{\mathbf{i}}$, doing the same for perturbation pressure from wave motion (not in static equilibrium), $p=P+p_{i}$, and subtracting the equations governing the barotropic flow, as does Baines [1982], yields a perturbation equation of motion

$$
\frac{\partial \mathbf{u}_{\mathbf{i}}}{\partial t}+\mathbf{f} \times \mathbf{u}_{\mathbf{i}}+\frac{\nabla p_{i}}{\bar{\rho}_{o}}+\frac{\rho_{i} g \hat{\mathbf{z}}}{\bar{\rho}_{o}}=\mathbf{B}-\left(\mathbf{u}_{\mathbf{i}} \cdot \nabla \mathbf{u}_{\mathbf{i}}+\mathbf{U} \cdot \nabla \mathbf{u}_{\mathbf{i}}+\mathbf{u}_{\mathbf{i}} \cdot \nabla \mathbf{U}\right)
$$



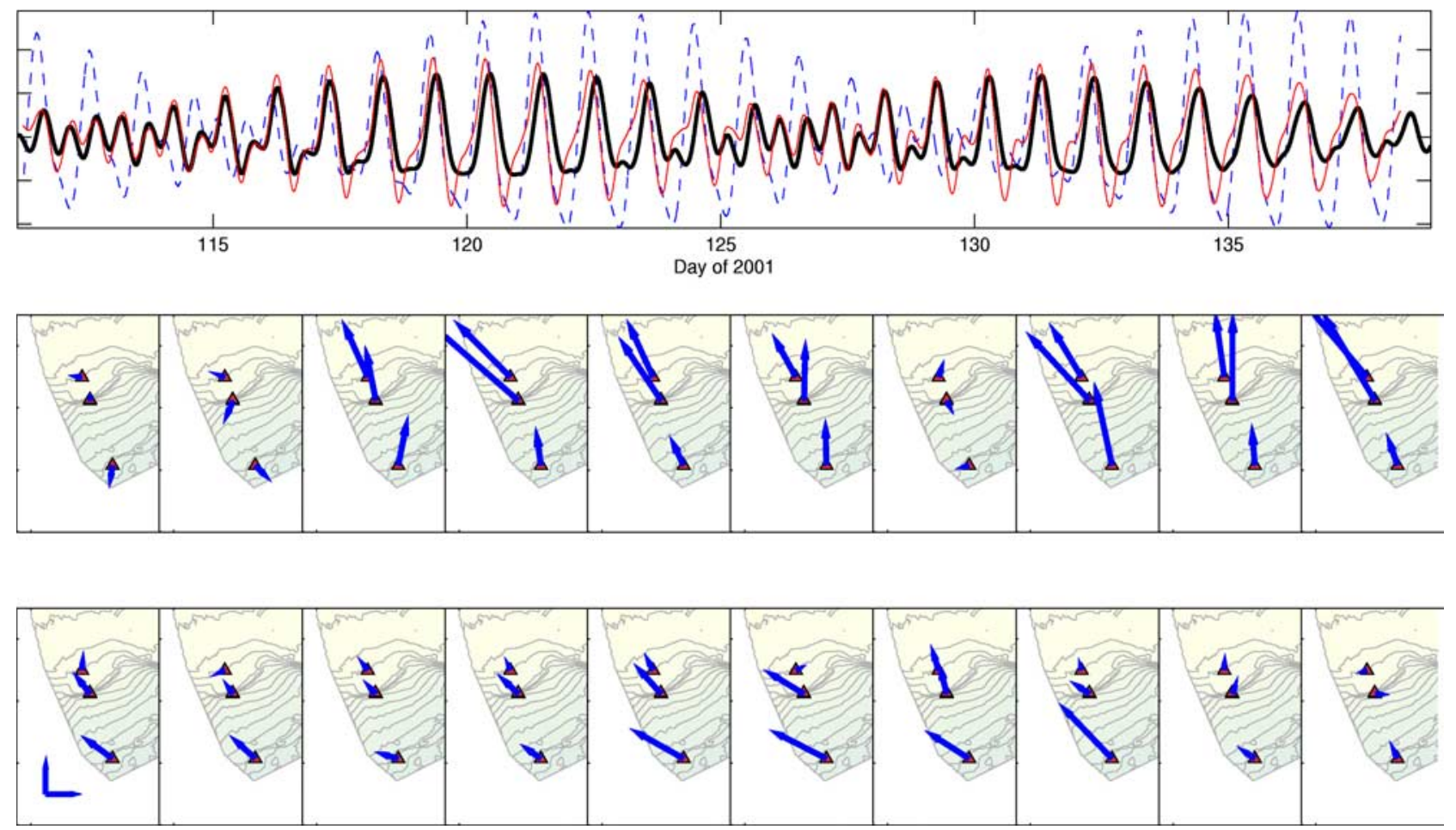

Figure 9. Internal tide energy flux vectors are shown. (bottom) Semidiurnal band flux vectors taken at the center of 10 equal length time windows are plotted on their respective locations (S7 (lowest), S5, and S4). The scale arrows at the lower left show $1000 \mathrm{~W} / \mathrm{m}$ east and north flux. The ten time windows correspond with those of Figure 8. (middle) Diurnal flux vectors are shown with the same scaling. (top) Three time series for comparison: in dashed blue, the harmonically fitted internal tidal displacement of the $20^{\circ} \mathrm{C}$ isotherm at S5, from Duda et al. [2004], and in thick black and thin red, the harmonically fitted northward barotropic tidal currents at S7 and S5, respectively, from Beardsley et al. [2004]. The $y$ axis range is [-21 29].

Here, $g$ is the gravitational acceleration, $\rho_{o}$ is the density of an unstratified ocean having the same surface tidal displacement as the local actual ocean, $\rho_{i}$ is the density perturbation of the internal tide, $\mathbf{f}$ is twice the local vertical projection of Earth's angular velocity, and $\mathbf{B}$ is a forcing term described in the next paragraph. This is the equation of Baines [1982] and earlier papers with three supplementary nonlinear terms on the right-hand side.

[51] The linear term $\mathbf{B}$ is a density perturbation that can drive internal tides, sometimes called the Baines force or the body force [Garrett and Gerkema, 2007]. It is often written in a form that is proportional to the seafloor slope [Carter et al., 2006; Gerkema, 2001; Nash et al., 2006]. It has the form

$$
\mathbf{B}=\frac{-g \rho_{s}}{\rho_{o}} \hat{\mathbf{z}}
$$

where $\rho_{s}$ is the density perturbation caused by the barotropic motion. This force acts only in the vertical, and is related to barotropic vertical velocity via $\partial \rho_{s} / \partial t=-U_{z} \partial \rho_{o} / \partial z \propto U_{z} N^{2}$. Its spatial and temporal structure is determined by the flow of three-dimensional barotropic tidal currents over complex bathymetry. An accurate 3-D map of $\rho_{s}$ (and thus B) may be computable from detailed knowledge of bathymetry and barotropic transport (i.e., from a computational flow simulation). A less accurate version can be computed by solving Laplace's tidal equations (or long-wave equations) with less detailed bathymetry. It is important to note that the temporal differentiation means that $\mathbf{B}$ has the tidal frequency in the denominator, so diurnal forcing would be double semidiurnal forcing, all else being equal (current amplitude, for instance). This is because $\mathbf{B}$ is the density or buoyancy perturbation and is given by the integral of vertical velocity, which for a given amplitude has a value proportional to period.

[52] $\mathbf{B}$ is not a function of the slope of the bottom relative to the slope of internal wave characteristics, which is a frequency-dependent quantity. Therefore maps of $\mathbf{B}$ would not identify the strong internal tide generation expected for near-critical bathymetry. The stream function form of the linear baroclinic response equation includes the second spatial derivative of the depth [Baines, 1982; Garrett and Gerkema, 2007], also not equal to the slope.

[53] Although B is usually the largest of the three forcing terms on the right-hand side of (3), it has only a vertical component. As a consequence, the only forcing terms in the horizontal momentum equations are the nonlinear ones. Thus the importance of the nonlinear terms may be best measured not with respect to $\mathbf{B}$, but with respect to other terms in the horizontal equations. One such term is the Coriolis term, known to be important for internal waves in the tidal band, causing the well-known flat elliptical orbits of low-frequency internal waves. 
[54] The magnitude of the first two nonlinear terms is as large as $7 \times 10^{-6} \mathrm{~m} \mathrm{~s}^{-2}$ for ASIAEX, computed via $a_{o}^{2} k$, where $k$ is the horizontal wave number of the diurnal internal tide, approximately $2 \pi \times 10^{-5} \mathrm{~m}^{-1}$, and $a_{o}=0.25$ is current amplitude. For a $0.25 \mathrm{~m} / \mathrm{s}$ current, the Coriolis term magnitude at latitude $22^{\circ}$ is $1.4 \times 10^{-5}$, only two times as large as the nonlinear term. For comparison, B appearing in the vertical equation is computed as follows: A $3400-\mathrm{m}$ diurnal excursion ellipse is multiplied by $\tan \left(0.25^{\circ}\right)$ to obtain a vertical excursion of $15 \mathrm{~m}$ near the seafloor. Multiplication of this by the density gradient gives density anomalies near 0.1 near the seafloor. This gives a $\mathbf{B}$ magnitude of about $10^{-3}$. This is significantly greater than the nonlinear terms, but appears only in the vertical equation.

[55] The magnitude of the third nonlinear term $\mathbf{u}_{\mathbf{i}} \cdot \nabla \mathbf{U}$ can also be nonnegligible because the gradient of $\mathbf{U}$ can be high in sloping areas. The components of $\nabla \mathbf{U}$ measured between $\mathrm{S} 5$ and $\mathrm{S} 4$, a 9-km span, are typically about $4 \mathrm{~cm} / \mathrm{s}$ reaching as high as $10 \mathrm{~cm} / \mathrm{s}$, giving typical and maximum term sizes of 1 to $3 \times 10^{-6}$ for $\mathbf{u}_{\mathbf{i}}$ of $25 \mathrm{~cm} / \mathrm{s}$. This is one tenth the Coriolis term.

[56] The high energy in the internal tide overtones (evident from the sawtoooth waveform in Figure 2 (bottom)) demonstrates the nonlinearity of internal tide propagation in this region (i.e., the importance of the $\mathbf{u}_{\mathbf{i}} \cdot \nabla \mathbf{u}_{\mathbf{i}}$ term). Because the internal and barotropic tides in the study area have comparable current speeds, the second nonlinear term $\left(\mathbf{U} \cdot \nabla \mathbf{u}_{\mathbf{i}}\right)$ should also play a role.

[57] This is not the first modified form of the Baines equation to appear in the literature. The effect of the locally horizontal components of the Earth's rotation was found to increase barotropic to baroclinic energy conversion by $2 \%$ for chosen seafloor and tide potential examples, and energy was found to travel along characteristics not parallel to simple internal wave characteristics [Baines and Miles, 2000]. This effect was also examined by Gerkema and Shrira [2006]. Another study did not involve modifications to the equations, but rather pertained to the nonlinearity of the problem. That study examined departure of forcing from tidal harmonic sinusoids caused by nonlinear processes. That departure was suggested to be a cause of increased bandwidth and spatial beam width of internal tides even with linear barotropic/baroclinic conversion [van Haren, 2006].

[58] The $\partial \mathbf{u}_{\mathbf{i}} / \partial t$ and the $\mathbf{u}_{\mathbf{i}} \cdot \nabla \mathbf{u}_{\mathbf{i n}}$ terms together form the shock wave equation. The behavior of this equation is discussed by Farmer [1978]. Disregarding the effect of the other terms, the fact that $\mathbf{U} \cdot \nabla \mathbf{u}_{\mathbf{i}}$ is also nonnegligible means that parcels can be accelerated by the barotropic flow as they would be by nonlinear self interaction. Spatial variation of $\mathbf{U}$ and $\nabla \mathbf{u}_{\mathbf{i}}$ will give spatial variability to the term, creating spatial structure in the baroclinic response field. One possible overall behavior is initiation of an internal tide via the effects of $\mathbf{B}$, with the Coriolis effect keeping $\mathbf{u}_{\mathbf{i}}$ aligned with a cyclonically rotating tidal $\mathbf{U}$, thus allowing continual reinforcing influence of the nonlinear terms. If $\mathbf{U}$ and $\mathbf{u}_{\mathbf{i}}$ were not matched, however, then the nonlinear forcing would be reduced, or even counteracted under certain conditions (section 5.4).

[59] The final nonlinear term, $\mathbf{u}_{\mathbf{i}} \cdot \nabla \mathbf{U}$, is perhaps the most interesting term because a similar term appears in
Reynold's equations. This is the term responsible for the production term in the turbulent kinetic energy budget equation, which is the Reynold's stress times the background shear [Osborn, 1980]. The action of this term in the horizontal momentum equation may similarly produce baroclinic response.

[60] The nonlinear terms contain $\mathbf{u}_{\mathbf{i}}$ and as such they cannot be explicitly written as source terms fully described in terms of the barotropic tide. This inability to separate the barotropic and baroclinic equations, as done by Baines (whose approach is a single-scatter type of approximation), has in part led to the use here of pictorial descriptions of the processes at work (Figures 8 and 9).

\subsection{Slope Criticality}

[61] The issue of criticality of the bottom slope deserves a closer look. The main point of this section is that criticality is important not only near the bottom, but over the entire water column, and must be appropriately evaluated.

[62] Linear internal wave particle orbits are ellipses lying in planes parallel with characteristics, with minor to major axis ratios $f / \omega$. Harmonic currents having trajectories of this type, for example caused by deflection of surface tidal current over a suitably formed bump, would be free internal waves and would propagate energy along characteristics.

[63] The fact that the group velocity is aligned with characteristics, which have slopes defined by $f, N$ and $\omega$, gives rise to interesting effects upon (nonspecular) reflection from near-critical slopes, including large energy density and probable instability [Eriksen, 1985; Gilbert and Garrett, 1989]. Intuitively then, critical slopes should have a strong effect on generation because characteristics would remain parallel with the seafloor over a lengthy domain, or nearly so, and energy would not propagate vertically away from a strong generation zone at constant height above bottom.

[64] Over a sloped seafloor, barotropic tidal transport causes depth-dependent currents with tilt varying linearly from horizontal at the surface to a value parallel to the seafloor at the seafloor [Baines, 1982]. For the case of a bottom slope equal to the slope of internal wave characteristics (at a given frequency) computed for $N$ at the seafloor, barotropic current particle orbits (at the same frequency) with the correct major-to-minor axis ratio, and with major axis aligned with the seafloor gradient, would satisfy internal free wave conditions at the seafloor.

[65] Note that forcing of internal waves is not confined to the near-bottom region. Typically, the stratification increases with height above the bottom, bending $c$ slope toward horizontal, in the same manner that barotropic current tilt decreases linearly. The two effects are not totally synchronized, however. For a supercritical seafloor slope and finite stratification, there is likely to be at least one depth where the tilt of the barotropic current matches $c$.

[66] The degree to which forced particle orbits are inclined to match internal wave orbits can be determined using a depth-adjusted criticality parameter $\gamma=\left|s_{a}(z) / c(z)\right|$, where $s_{a}=s \times(-z / H)$ is zero at the surface and equal to the seafloor slope $s=|\mathbf{s}|$ at the seafloor. Barotropic flows deflected by a sloping seafloor are generally modeled to be parallel to $s_{a}$. Zones of the water column where $\gamma$ is near 


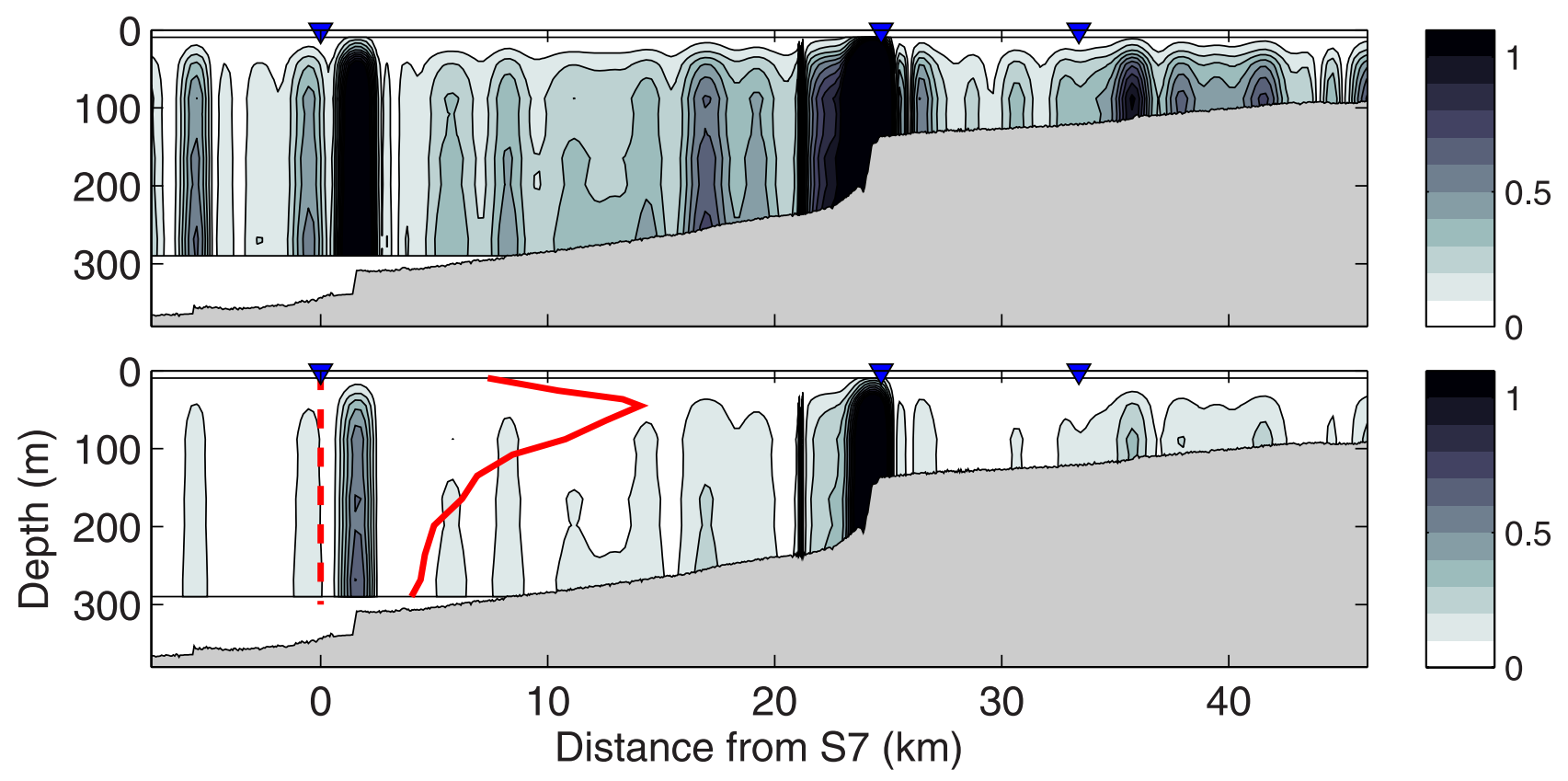

Figure 10. Contours of $\gamma=\left|s_{a} / c\right|$ for (top) diurnal and (bottom) semidiurnal bands. Supercritical areas are not contoured above one and are shown in black. Buoyancy frequency $N(z)$ used to compute $c(z)$ is shown in the lower frame (cycles per hour). A low-passed version of the bathymetry that is plotted is used for calculation. The triangles show positions of (from left) S7, S5, and S4.

one would be expected to be source regions for internal tides.

[67] Figure 10 shows $\gamma$ in a slice along the mooring line for diurnal and semidiurnal frequencies. $N(z)$ is shown in the lower (semidiurnal) frame, derived from CTD casts. The contour interval is 0.1 . White areas have $\gamma<0.1$.

[68] For both species, $\gamma$ is supercritical at the steps (shown in black), so the steps are unlikely to cause strongly differing response between the species. In the semidiurnal case, the parameter is close to zero (greatly subcritical) in the majority of the slice. In the diurnal case, large zones have values between 0.3 and 0.7 (between 8 - and 22-km distance in the plot). These tend to be deeper in the water column where $s_{a}(z)$ is larger. Beneath the $N^{2}$ maximum $s_{a}$ and $c$ both decrease with height, so $\gamma$ changes slowly. Above the $N^{2}$ maximum, effects of $c(z)$ increasing upward in the water column tend to counteract the depth scaling within $s_{a}$, but do not cause $\gamma$ to increase enough to rival the bottom enhancement.

[69] Almost the entire section is highly subcritical in the semidiurnal band $(\gamma \ll 1)$, and subcritical to a lesser degree in the diurnal band. The supercritical isolated steep areas have thin narrow surfaces of criticality on either side of the supercritical zones. The observed strong diurnal internal tides may indicate that the diurnal values of $0.3<\gamma<0.7$ are conducive to generation.

[70] Alternative diagnostic parameters would be $\gamma$ times the magnitude of $\mathbf{B}$, or $\gamma$ times the barotropic tide power $\langle\mathbf{B} \cdot \mathbf{U}\rangle$, where the brackets indicate average over one cycle. Tide power has been plotted in a vertical plane to identify forcing areas [Nash et al., 2006], but this alone does not include effect of slope criticality. Likewise, others have looked at the ratio of bottom slope to characteristic slope (a component of our $\gamma$ ) along track lines [Martin et al., 2006] or over spatial areas. The alternative product parameters would combine criticality and forcing power, although in an ad hoc way. Better combinations of these factors may arise from future work.

\subsection{Horizontally Coherent Forcing}

[71] An important situation occurs when the bottom slope is near critical over a long distance upslope (many kilometers), giving rise to spatially coherent forcing of internal waves in the propagation direction. This is the situation near the ASIAEX seafloor for the diurnal band. The flux data show the expected strong response. Appealing to the arguments of the two prior sections, forcing mechanisms mentioned there would act uniformly over extended spatial domains, so that forcing would be fairly uniform along characteristics. This is important because internal waves have constant phase along characteristics. To illustrate, diurnal characteristics in Figure 6 (top) would not cross in and out of regions of widely differing $\gamma$ (Figure 10) between S7 and S5.

[72] On the other hand, there are limits to the size of a (finite) source region that can coherently force waves. A practical limit for the maximum effective forcing domain size is one sixth of a wavelength (equivalent to one radian of phase difference for waves coming from opposite sides of the patch). In this situation all the energy going into the internal tide can form a propagating wave, with no interference, (i.e., the source region of diameter $a$ defined by the set of positions $r_{s}$ is small enough so that phase of the exponential in the resultant wave at position $\hat{r}, p(\hat{r})=\sum r_{s}$ $\exp \left\{i\left[\omega t-k_{h} \cdot\left(\hat{r}-r_{s}\right)\right]\right\}$, is approximately constant; the practical limit is defined by $k_{h} a=1$ [Rayleigh, 1945, chap. XVII] Internal wave horizontal wavelengths are much shorter than surface tide wavelengths, and may be compa- 
rable in size to generation regions, causing this limit to potentially come into play. Phase coherent forcing from very long barotropic waves over an evenly sloped seafloor spanning large fractions of an internal wave wavelength would not allow efficient generation.

[73] All of the forcing terms in (3) have spatial variability that must be considered in this way. The scale lengths of $\mathbf{U}$ and $\mathbf{u}_{\mathbf{i}}$ will control the effect of the nonlinear terms. Coherent forcing also applies to the buoyancy forcing $\mathbf{B}$ because spatial maps of $\mathbf{B}$ may exhibit scales approaching or exceeding wavelengths of internal waves (tens of kilometers). The Green's function methodology of Cox and Sandstrom [1962] allows for the effects of forcing coherency in a mathematically comprehensive way for the linear situation, and may be adaptable to solution of (3).

[74] Incoherent forcing (cancelation) due to large $k_{h} a$ is less probable for diurnal internal tides than semidiurnal because the diurnal waves have longer wavelengths. Assuming the 1-radian limit for coherent forcing, barotropic flow forcing with spatially constant phase will begin to undergo cancelation at $6 \mathrm{~km}$ for a semidiurnal wave, and 17 $\mathrm{km}$ for a diurnal wave. (Constant forcing phase is a good approximation, depicted in Figure 3. Phase numbers are given by Beardsley et al. [2004]; the dominant constituent phases vary by at most $50^{\circ}$ from S7 to S4.) Because the slope is uniform except for the steps (Figure 10), the size of the forcing region has the potential to exceed the coherent length limit for each species. Figure 10 also shows that slightly subcritical conditions (which the flux results indicate to be favorable for generation) exist in the diurnal band, with variable criticality, for tens of kilometers up the slope. In summary, the large spatial scales of forcing mean that the longer diurnal internal waves are more likely to effectively radiate.

\section{Summary}

[75] Time series of internal tidal energy flux at four positions, three near the continental shelf edge, and one further offshore of the shelf edge, provide evidence of more efficient generation of diurnal internal tides than semidiurnal internal tides. In particular, the flux of diurnal baroclinic energy onto the shelf exceeds that measured at the deeper location, whereas semidiurnal energy and energy flux decline monotonically up the slope. The diurnal internal tides penetrate further onto the shelf than semidiurnal counterparts observed in the Australian North West Shelf [Holloway et al., 2001]. Complete flux results are given in Table 1 and Figure 5.

[76] Temporal and spatial variability of the fluxes and the nonalignment of internal tide flux vectors adds ambiguity to the interpretation. Future experiments should include a twodimensional array of moorings spanning both the alongshelf and cross-shelf directions to better measure energy flux horizontal convergence and divergence, in the manner of a recent short experiment [Chang et al., 2006].

[77] Ratios of diurnal to semidiurnal energy flux and kinetic energy exceed estimates of the ratio of barotropic forcing in the diurnal and semidiurnal bands. The ratio of diurnal to semidiurnal internal tidal energy flux onto the shelf at position S4 is approximately eleven to one, which exceeds the ratio of barotropic kinetic energies in the two species by a factor of three. It also exceeds the ratio of diurnal to semidiurnal forcing $\left\langle U_{z} \mathbf{B}\right\rangle$ [Nash et al., 2006], evaluated at S7 and S5 using $U^{2}$ values from Table 1 and the ratio of periods, by the same factor. The apparent strong diurnal response may be explained by the shallow incline of the slope (somewhat diurnal subcritical), the long wavelength of diurnal waves (good response to spatially coherent forcing), and frequently diurnally rotating surface tide particle trajectories. If the assumption is made that the semidiurnal flux is incident from deeper water and is not the result of local conversion of barotropic tidal energy, the logical conclusion is that no semidiurnal internal tides are generated, and the diurnal/semidiurnal discrepancy is even more striking.

[78] The ratio of diurnal to semidiurnal barotropic energy flux (forcing) is roughly 2.5 (Table 1); the ratio of mean squared currents is about 3; and the ratio of maximum diurnal to semidiurnal barotropic current (considered independently) is also roughly 3 (Figure 5), so that the ratio of peak kinetic energies is 9 . The weaker forcing might explain weaker generation of semidiurnal species, but not a lack of generation in a linear context.

[79] The irregular details of the internal tide kinetic energy and energy flux time series, when compared to the regularity of the tide, illustrate the complicated nature of internal tide generation. The varying baroclinic response to the steady tidal forcing is striking. The stratification is relatively stable, so the variability is not trivially explained by temporal changes in the characteristic slopes $c(x, y, z, \omega)$. On the other hand, subtidal currents and shears are time-dependent, possibly contributing to the variability. Interference from the incident internal tides on the local generation process is another possible complicating effect. Such incident waves would alter the phase relationship between the two nonlinear forcing factors, would alter the phase relationship between the linear forcing term and the nonlinear terms, and may interfere with the linear forcing.

[80] Although the field results are the major emphasis of the paper, the possible roles of two factors related to the barotropic wave particle velocity vector in the generation process were also examined. The factors are internal wave orbital regularity, and proximity of orbital inclination to the slope of characteristics. A third factor, coherence of forcing, measured using the baroclinic wavelength, was also examined.

[81] Considering the first factor, the relationship between barotropic tidal flow trajectories and observed baroclinic response was examined graphically. Observed temporal behavior of internal tides appears to be linked to barotropic wave particle orbits. Diagrams of barotropic tidal particle orbits suggest that simple elliptical particle orbits provide the strongest baroclinic response, matching intuition. This intuition, plus the intuition that barotropic tidal velocities matching baroclinic internal wave orbits (including orbit inclinations from the horizontal, possible at the seafloor for critical slope and, for example, halfway to the surface for a slope twice critical) does not show up in linearized equations [Baines, 1982, and references therein], but is represented in a nonlinear diagnostic equation. The magnitudes of nonlinear terms in the nonlinear diagnostic momentum equation involving the velocity vector were examined. Effects from these terms may eventually more rigorously explain the observed internal tide complexity. 
[82] Diurnal barotropic particle orbit planforms, and orbit inclinations, are well matched to theoretical diurnal internal wave orbits, leading to a very strong internal tidal response. The orbit matches are not as good in the semidiurnal band as in the diurnal. This may explain why the semidiurnal response is weaker and may be nonexistent.

[83] The next factor, particle orbit inclination with respect to characteristic inclination, was examined using $\gamma$, a depthadjusted measurement of seafloor slope with respect to characteristic slope. This parameter was closest to one for the diurnal band, possibly explaining the enhanced diurnal internal wave response, and offering another explanation for the apparently weak semidiurnal internal tide generation in the mooring region.

[84] The effect of source region size in comparison to radiating wavelength (factor three), a familiar notion in transducer engineering, antenna theory, and scattering theory, also favors diurnal internal tides over semidiurnal because of the longer diurnal wavelength.

[85] The approaches taken here emphasize the fact that internal waves are forced throughout the entire water column, not only at the seafloor. They also emphasize the fact that barotropic tides may impart momentum to internal waves, that nonlinearity may play a role, and that oceanic internal tide details may be inconsistent with the response computed using only surface tide buoyancy forcing.

[86] Acknowledgments. This work was funded by grants from the U.S. Office of Naval Research, Physical Oceanography, and Ocean Acoustics programs and by the Woods Hole Oceanographic Institution. LR acknowledges support from the Doherty Foundation.

\section{References}

Alford, M. H., and Z. Zhao (2007), Global patterns of low-mode internalwave propagation, part I: Energy and energy flux, J. Phys. Oceanogr., 37, 1829-1848, doi:10.1175/JPO3085.1.

Baines, P. G. (1982), On internal tide generation models, Deep Sea Res., Part A, 29, 307-338.

Baines, P. G., and J. W. Miles (2000), On topographic coupling of surface and internal tides, Deep Sea Res., Part I, 47, 2395-2403.

Beardsley, R. C., T. F. Duda, J. F. Lynch, J. D. Irish, S. R. Ramp, C.-S. Chiu, T. Y. Tang, Y.-J. Yang, and G. Fang (2004), Barotropic tide in the northeast South China Sea, IEEE J. Oceanic Eng., 29, 1075-1086.

Carter, G. S., M. C. Gregg, and M. A. Merrifield (2006), Flow and mixing around a small seamount on Kaena Ridge, Hawaii, J. Phys. Oceanogr., $36,1036-1052$.

Chang, M.-H., R.-C. Lien, T. Y. Tang, E. A. D’Asaro, and Y. J. Yang (2006), Energy flux of nonlinear internal waves in northern South China Sea, Geophys. Res. Lett., 33, L03607, doi:10.1029/2005GL025196.

Cox, C. S., and H. Sandstrom (1962), Coupling of internal and surface waves in water of variable depth, J. Oceanogr. Soc. Jpn., 18, 499-513.

Duda, T. F., J. F. Lynch, J. D. Irish, R. C. Beardsley, S. R. Ramp, C.-S. Chiu, T. Y. Tang, and Y.-J. Yang (2004), Internal tide and nonlinear internal wave behavior at the continental slope in the northern South China Sea, IEEE J. Oceanic Eng., 29, 1105-1130.

Egbert, G. D., A. F. Bennett, and M. G. G. Foreman (1994), TOPEX/ POSEIDON tides estimated using a global inverse model, J. Geophys. Res., 99, 24,821-24,852.
Eriksen, C. C. (1985), Implications of ocean bottom reflections for internal wave spectra and mixing, J. Phys. Oceanogr., 15, 1145-1156.

Farmer, D. M. (1978), Observations of long nonlinear internal waves in a lake, J. Phys. Oceanogr., 8, 63-73.

Garrett, C., and T. Gerkema (2007), On the body-force term in internal-tide generation, J. Phys. Oceanogr., 37, 2172-2175.

Gerkema, T. (2001), Internal and interfacial tides: Beam scattering and local generation of solitary waves, J. Mar. Res., 59, 227-255.

Gerkema, T., and V. I. Shrira (2006), Non-traditional reflection of internal waves from a sloping bottom, and the likelihood of critical reflection, Geophys. Res. Lett., 33, L06611, doi:10.1029/2005GL025627.

Gilbert, D., and C. Garrett (1989), Implications for ocean mixing of internal wave scattering off irregular topography, J. Phys. Oceanogr., 19, $1716-1729$

Holloway, P. E., and M. A. Merrifield (2003), On the spring-neap variability and age of the internal tide at the Hawaiian Ridge, J. Geophys. Res., 108(C4), 3126, doi:10.1029/2002JC001486.

Holloway, P. E., P. G. Chatwin, and P. Craig (2001), Internal tide observations from the Australian North West Shelf in summer 1995, J. Phys. Oceanogr., 31, 1182-1199.

Levine, M. D., and T. J. Boyd (2006), Tidally forced internal waves and overturns observed on a slope: Results from HOME, J. Phys. Oceanogr., 36, 1184-1201.

MacKinnon, J. A., and M. C. Gregg (2003), Mixing on the late-summer New England shelf: Solibores, shear and stratification, J. Phys. Oceanogr., 33, 1476-1492.

Martin, J. P., D. L. Rudnick, and R. Pinkel (2006), Spatially broad observations of internal waves in the upper ocean at the Hawaiian Ridge, J. Phys. Oceanogr., 36, 1085-1103.

Nash, J. D., E. Kunze, J. M. Toole, and R. W. Schmitt (2004), Internal tide reflection and turbulent mixing on the continental slope, J. Phys. Oceanogr., 34, 1117-1134.

Nash, J. D., E. Kunze, C. M. Lee, and T. B. Sanford (2006), Structure of the baroclinic tide generated at Kaena Ridge, Hawaii, J. Phys. Oceanogr., 36, $1123-1135$

Osborn, T. R. (1980), Estimates of the local rate of vertical diffusion from dissipation measurements, J. Phys. Oceanogr., 10, 83-89.

Pawlowicz, R., R. Beardsley, and S. Lentz (2002), Classical tidal harmonic analysis including error estimates in MATLAB using T_TIDE, Comput. Geosci., 28, 929-937.

Pingree, R. D., and A. L. New (1991), Abyssal penetration and bottom reflection of internal tidal energy in the Bay of Biscay, J. Phys. Oceanogr., 21, 28-39.

Rainville, L., and R. Pinkel (2006), Baroclinic energy flux at the Hawaiian Ridge: Observations from the R/P FLIP, J. Phys. Oceanogr., 36, $1104-1122$

Ramp, S. R., T. Y. Tang, T. F. Duda, J. F. Lynch, A. K. Liu, C.-S. Chiu, F. L. Bahr, H.-R. Kim, and Y.-J. Yang (2004), Internal solitons in the northeastern South China Sea. part I: Sources and deep water propagation, IEEE J. Oceanic Eng., 29, 1157-1181.

Rayleigh, J. W. S. (1945), The Theory of Sound, vol. 2, 504 pp., Dover, New York.

Robertson, R. (2006), Modeling internal tides over Fieberling Guyot: Resolution, parameterization, performance, Ocean Dyn., 56, 430-444.

Schock, S. G. (2004), Remote estimates of physical and acoustic sediment properties in the South China Sea using chirp sonar data and the Biot model, IEEE J. Oceanic Eng., 29, 1218-1230.

van Haren, H. (2006), Nonlinear motions at the internal tide source, Geophys. Res. Lett., 33, L11605, doi:10.1029/2006GL025851.

T. F. Duda, Applied Ocean Physics and Engineering Department, Woods Hole Oceanographic Institution, Woods Hole, MA 02543, USA. (tduda@ whoi.edu)

L. Rainville, Applied Physics Laboratory, University of Washington, 1013 NE 40th Street, Seattle, WA 98105-6698, USA. 